\title{
Robótica Educacional e Formação de Professores: uma Revisão Sistemática da
}

\section{Literatura}

João Joaquim de Freitas Neto, IFRS/MPIE Campus Porto Alegre, joao.freitas@gmail.com - Orcid: https://orcid.org/0000-0001-8361-6991

Silvia de Castro Bertagnolli, IFRS/MPIE Campus Porto Alegre, silvia.bertagnolli@poa.ifrs.edu.br - Orcid: https://orcid.org/0000-0001-7495-6636

Resumo: A Robótica educacional (RE) é um recurso pedagógico que permite desenvolver diversas habilidades, além de possibilitar a integração do pensamento computacional as mais variadas áreas do conhecimento. Entretanto, para que possa ser usada como abordagem em sala de aula é necessário que o professor esteja devidamente qualificado e de posse de uma proposta pedagógica condizente com a realidade na qual ele está inserido. Assim, esse artigo tem como objetivo apresentar uma revisão sistemática de publicações direcionadas à formação de docentes para usarem a robótica educacional. Como resultados destacam-se a escassez de trabalhos que abordam a utilização de kit de robótica livre na formação de docentes, estratégias de baixo custo na implementação da RE nas escolas e a necessidade de sua implementação no currículo escolar.

Palavras-chaves: Formação docente, Robótica educacional, Educação básica.

\section{Educational Robotics and Teacher Training: A Systematic Literature Review}

Abstract: Educational robotics (ER) is a pedagogical resource that allows the development of various skills, in addition to enabling the integration of computational thinking in the most varied areas of knowledge. However, to be used as a classroom approach, it is necessary that the teacher is properly qualified and with knowledge of a pedagogical proposal consistent with the reality in which he is inserted. Thus, this article aims to present a systematic review of publications aimed at training teachers to use educational robotics. Among the results found, we highlight the limited number of studies that address the use of robotics free kit in teacher training, low-cost strategies in the implementation of RE in schools and the need for its implementation in the school curriculum.

Keywords: Teacher training, Educational robotics, Basic education.

\section{Introdução}

A Base Nacional Curricular Comum (BNCC) ressalta a importância do uso da tecnologia na escola e o papel fundamental dos educadores no processo de qualificação dos educandos para um uso ético das diversas tecnologias existentes. Ela aponta, ainda, a importância do uso de várias estratégias no desenvolvimento do raciocínio, privilegiando o questionamento, a análise e a busca por soluções críticas e inovadoras (Brasil, 2017).

Dentro desta perspectiva, a Robótica Educacional (RE) pode ser vista como uma ferramenta capaz de trazer à escola uma maior aproximação com o mundo tecnológico, ao passo que estimula os estudantes a desenvolverem as competências previstas na BNCC; e viabiliza que os aprendizes possam compreender o mundo digital de uma forma diferenciada, com ela é possível "mostrar exatamente como uma entrada é capturada [...], processada, executada e devolvida para quem 'comanda"” (Silva; Blikstein, 2020). 
Nesse contexto a robótica educacional pode contribuir de forma significativa como ferramenta pedagógica para auxiliar os professores a promoverem a construção do pensamento crítico, científico e criativo, sobretudo em escolas públicas. Conforme argumenta Ribeiro (2006), a robótica educacional ainda não avançou significativamente no que diz respeito a sua utilização. Entretanto, para traçar estratégias que viabilizem o uso dessa ferramenta, em sala de aula, por parte dos docentes, é necessário primeiro entender de que forma a robótica educacional está presente nas práticas pedagógicas docentes atuais. Através dessa revisão sistemática da literatura, acredita-se que será possível reunir informações que sirvam de referência para nortear futuros estudos e fomentar debates que auxiliem na formação e na prática dos educadores.

Para o desenvolvimento deste trabalho, selecionou-se como método de pesquisa a RSL - uma revisão sistemática da literatura, que consiste em examinar e analisar estudos publicados considerando um tópico de pesquisa específico, e que tem por intensão verificar as evidências disponíveis. Portanto, ela tem como objetivo identificar e classificar a pesquisa relacionada a um tópico amplo de pesquisa (Kitchenham; Charters, 2007). Assim, o presente mapeamento sistemático pretende não só identificar trabalhos relacionados ao assunto robótica educacional, mas apontar possibilidades de pesquisas futuras.

Nas próximas seções deste artigo o percurso metodológico (seção 2) define as etapas de revisão e seu desenvolvimento, a análise e discussão (seção 3) relaciona a construção dos resultados obtidos durante o processo, seguido pelas considerações finais (seção 4) obtidas ao término da investigação.

\section{Revisão Sistemática da Literatura}

De acordo com Kitchenham, Brereton e Budgen (2011) o resultado de uma RSL pode ajudar a identificar lacunas na área em que foi realizado, possibilitando assim um melhor posicionamento de novas atividades de pesquisa. Para tanto, Petersen et al. (2008) divide o processo de revisão da literatura em cinco etapas: definição das questões de pesquisa, execução da pesquisa, triagem dos documentos obtidos, classificação dos documentos e extração de dados, e mapeamento.

Desse modo, o primeiro passo deste trabalho compreendeu definir as questões de pesquisa, as quais nortear a presente investigação e a análise de cada estudo analisado (Quadro 1). As questões apresentadas trazem o propósito de delinear a busca e apresentar o objetivo da revisão, tendo em vista que este trabalho é parte de uma pesquisa mais ampla que está em andamento, e na qual os autores estudam a robótica educacional com foco na formação de docentes. Observa-se que as questões elaboradas tentam identificar estratégias usadas na formação docente, áreas do conhecimento abordadas, níveis de ensino e recursos tecnológicos, de modo a identificar alternativas possíveis de serem adotadas em escolas da rede pública de ensino.

\section{Quadro 1 - Questões de pesquisa para investigar}

1. As formações são direcionadas para a área de conhecimento dos docentes?

2. Quais níveis de educação básica costumam estar associados a projetos de robótica educacional?

3. Quais são as plataformas de prototipagem utilizadas com mais frequência nas escolas da rede pública de educação básica? 
A busca dos estudos foi realizada utilizando-se como palavras-chaves, para pesquisas em repositórios brasileiros, (("formação docente" OR "formação professores" OR "capacitação docente" OR "capacitação professores" OR "qualificação docente" OR "qualificação professores") AND ("robótica educacional" OR "robótica educativa" OR "robótica pedagógica")). Já para repositórios internacionais usou-se ((“teacher training") AND ("educational robotics")). A partir dessas expressões de busca foram selecionados alguns repositórios de publicações científicas, nos quais a busca por estudos seria realizada.

Nesta revisão foram selecionados os repositórios de publicações apresentados no Quadro 2, a seleção desses repositórios foi realizada considerando a sua relevância para a área da Informática na Educação, no caso das quatro primeiras. Já o portal de teses e dissertações foi selecionado por agregar dissertações e teses de todas as regiões do país; e o portal de periódicos da Capes foi selecionado, pois ao acessar alguns repositórios internacionais percebeu-se que embora existissem publicações vinculadas ao tema o acesso era restrito, assim usando esse portal foi possível ter acesso às publicações constantes em repositórios internacionais. Salienta-se ainda que, o lapso de tempo delimitado para a busca das pesquisas compreendeu de janeiro de 2016 até março de 2021.

Quadro 2 - Repositórios utilizados na revisão da literatura

\begin{tabular}{|cl|l|}
\hline \multicolumn{1}{|c|}{ Repositórios } & \multicolumn{1}{c|}{ Endereço } \\
\hline 1. & $\begin{array}{l}\text { Portal de publicações da Comissão Especial de } \\
\text { Informática na Educação (CEIE) }\end{array}$ & $\begin{array}{l}\text { https://www.br- } \\
\text { ie.org/pub/index.php/index }\end{array}$ \\
\hline 2. & $\begin{array}{l}\text { Anais do Congresso Brasileiro de Informática } \\
\text { na Educação (CBIE), Anais do Workshop de } \\
\text { Informática na Escola (WIE) e Anais dos } \\
\begin{array}{l}\text { Workshops do Congresso Brasileiro de } \\
\text { Informática na Educação (WCBIE) - 2020 }\end{array}\end{array}$ & https://sol.sbc.org.br/index.php/cbie \\
\hline 3. & $\begin{array}{l}\text { Revista Novas Tecnologias na Educação } \\
\text { (RENOTE) }\end{array}$ & http://seer.ufrgs.br/renote/ \\
\hline 4. & $\begin{array}{l}\text { Revista Brasileira de Informática na Escola } \\
\text { (RBIE) }\end{array}$ & $\begin{array}{l}\text { http://www.br- } \\
\text { ie.org/pub/index.php/rbie }\end{array}$ \\
\hline 5. & Catálogo de Teses e Dissertações CAPES & http://catalogodeteses.capes.gov.br \\
\hline 6. & Portal de Periódicos CAPES & http://www.periodicos.capes.gov.br \\
\hline
\end{tabular}

Fonte: Elaborada pelos autores (2021).

As buscas foram realizadas utilizando-se as expressões apresentadas anteriormente e resultaram em 18 trabalhos em português e 48 em outros idiomas. Após, foram aplicados os critérios de inclusão e exclusão listados no Quadro 3, em dois processos de refinamento para os resultados obtidos, o primeiro pautado na leitura do título e do resumo; e o segundo por meio de leitura da introdução e da conclusão.

Quadro 3 - Critérios de inclusão e exclusão de documentos

\begin{tabular}{|c|c|}
\hline Critérios de Inclusão & Critérios de Exclusão \\
\hline $\begin{array}{l}\text { 1. Apresentam relação com a } \\
\text { educação. } \\
\text { 2. Apresentam abordagens } \\
\begin{array}{l}\text { práticas na formação de } \\
\text { docentes. }\end{array}\end{array}$ & $\begin{array}{l}\text { 1. Estudo que não apresenta relação com as questões de } \\
\text { pesquisa e objetivos dessa investigação. } \\
\text { 2. Estudo secundário - ou seja, outras revisões } \\
\text { sistemáticas da literatura e mapeamentos de trabalhos. } \\
\text { 3. Estudos que apresentam a robótica educacional sem } \\
\text { resultados e/ou validações. } \\
\text { 4. Estudos já identificados em outra(s) base(s). }\end{array}$ \\
\hline
\end{tabular}

Fonte: Elaborada pelos autores (2021).

Durante a leitura dos títulos e resumo dos trabalhos que retornaram da busca, observou-se que uma parcela considerável não trazia como foco a formação de docentes, reduzindo assim para quatro trabalhos obtidos em português e oito em língua estrangeira. 
Em sequência, realizou-se a análise da introdução e conclusão dos trabalhos, reduzindo o resultado a um total de 4 trabalhos em português e 6 em inglês, conforme esquematiza o Quadro 4. Por fim, após esses refinamentos foi realizada a leitura dos estudos na íntegra, visando obter uma melhor compreensão de como cada pesquisa foi conduzida, seus resultados e quais estratégias foram adotadas na formação dos docentes.

Quadro 4. Estudos encontrados no processo de busca

\begin{tabular}{|l|l|l|l|}
\hline Repositório & Resultado & $\mathbf{1}^{\circ}$ refinamento & $\mathbf{2}^{\circ}$ refinamento \\
\hline Portal CEIE & 0 & 0 & 0 \\
\hline CBIE, WIE e WCBIE - 2020 & 1 & 1 & 1 \\
\hline RENOTE & 0 & 0 & 0 \\
\hline RBIE & 0 & 0 & 0 \\
\hline Teses e Dissertações CAPES & 19 & 4 & 4 \\
\hline Periódicos CAPES & 47 & 7 & 5 \\
\hline Total & $\mathbf{6 7}$ & $\mathbf{1 2}$ & $\mathbf{1 0}$ \\
\hline
\end{tabular}

Fonte: Elaborada pelos autores (2021).

No portal de periódicos da Capes foram localizadas cinco publicações, sendo uma em periódico nacional (estudo E04) e quatro em periódicos internacionais: Technology, Knowledge and Learning, International Journal of Social Robotics, Education and Information Technologies and Journal of Science, Education and Technology, os quais também integram o repositório da Springer ${ }^{1}$.

Ao concluir os refinamentos foi possível um melhor entendimento das especificidades de cada estudo, bem como o cerne de cada pesquisa conduzida. O Quadro 5 elenca esses estudos, através de sua referência; e de sua identificação para uso nas próximas seções deste artigo.

\section{Quadro 5. Estudos científicos selecionados}

\begin{tabular}{|l|l|}
\hline Id & Referência de cada Estudo \\
\hline E01 & $\begin{array}{l}\text { SOUZA, I. M. L.; RODRIGUES, R. S.; ANDRADE, W. Introdução do Pensamento } \\
\text { Computacional na Formação Docente para Ensino de Robótica Educacional. In: } \\
\text { Congresso Brasileiro de Informática na Educação, 2016, Uberlândia. Anais... } \\
\text { Workshop do Congresso Brasileiro de Informática na Educação, Uberlândia: Sociedade } \\
\text { Brasileira de Computação, 2016, p. 1265-1274. Disponível em: https://www.br- } \\
\text { ie.org/pub/index.php/wcbie/article/view/7052. Acesso em: 22 jan. 2021. }\end{array}$ \\
\hline E02 & $\begin{array}{l}\text { ALIMISIS, D. Teacher Training in Educational Robotics: The ROBOESL Project } \\
\text { Paradigm. Technology, Knowledge and Learning, v. 24, p. 279-290, 2019. }\end{array}$ \\
\hline E03 & $\begin{array}{l}\text { Castro, E., Cecchi, F., Salvini, P. et al. Design and Impact of a Teacher Training Course, } \\
\text { and Attitude Change Concerning Educational Robotics. International Journal of } \\
\text { Society and Robotics, v. 10, p. 669-685, 2018. }\end{array}$ \\
\hline E04 & $\begin{array}{l}\text { PIEDADE, J. M. N. Interesse, conhecimento e autoconfiança de futuros professores e } \\
\text { professores em serviço no uso de robótica educacional em atividades de aprendizagem. } \\
\text { Educação \& Formação, v. 6, n. 1, p. 3345, jan., 2020. }\end{array}$ \\
\hline E05 & $\begin{array}{l}\text { EL-HAMAMSY, L.; CHESSEL-LAZZAROTTO, F.; BRUNO, B. et al. A computer } \\
\text { science and robotics integration model for primary school: evaluation of a large-scale } \\
\text { in-service K-4 teacher-training program. Education and Information Technologies, } \\
\text { v. 26, p. 2445-2475, 2020. }\end{array}$ \\
\hline E06 & $\begin{array}{l}\text { JAIPAL-JAMANI, K., ANGELI, C. Effect of Robotics on Elementary Preservice } \\
\text { Teachers' Self-Efficacy, Science Learning, and Computational Thinking. Journal of } \\
\text { Science, Education and Technology, v. 26, p. 175-192, 2017. }\end{array}$ \\
\hline E07 & $\begin{array}{l}\text { VARELA, C. D. A. A robótica educacional na escola indígena: inovaçães na } \\
\text { formação de professores. 2017. 78 f. Curitiba: Centro Universitário Internacional, } \\
\text { 2017. Dissertação de Mestrado Profissional em Educação e Novas Tecnologias. }\end{array}$ \\
\hline
\end{tabular}

${ }^{1}$ Disponível em: https://www.springer.com/. 


\begin{tabular}{|l|l|}
\hline E08 & $\begin{array}{l}\text { GREBOGY, E. C. Formação em Contexto de São José Dos Pinhais: Robótica } \\
\text { Sustentável. 2017. Curitiba: Centro Universitário Internacional, 2017. Dissertação em } \\
\text { Mestrado Profissional em Educação e Novas Tecnologias. }\end{array}$ \\
\hline E09 & $\begin{array}{l}\text { SILVA, J. F. S. Robótica Aplicada à Educação: uma análise do pensar e fazer dos } \\
\text { professores egressos do curso oferecido pelo município de João Pessoa-PB, } 2017 . \\
\text { Campina Grande: Universidade Estadual da Paraíba, 2017. Dissertação de Mestrado } \\
\text { Profissional em Formação de Professores. }\end{array}$ \\
\hline E10 & $\begin{array}{l}\text { ALMEIDA, P. C. T. A Arte de Aprender para Ensinar: discutindo a capacitação } \\
\text { de robótica com Arduino® para professores de ciências e matemática do município } \\
\text { de Paracambi/RJ, 2017. Nilópolis: Instituto Federal de Educação, Ciência e } \\
\text { Tecnologia do Rio de Janeiro, 2017. Dissertação de Mestrado Profissional em Ensino } \\
\text { de Ciências. }\end{array}$ \\
\hline
\end{tabular}

Fonte: Elaborada pelos autores (2021).

Após realizar a leitura dos trabalhos selecionados as questões de pesquisa foram revisitadas e cada uma delas foi analisada e respondida, como apresenta a próxima seção.

\section{Análise e Discussões}

Partindo-se da questão "As formações são direcionadas para a área de conhecimento dos docentes?" foi possível observar que as formações propostas para professores, em sua grande maioria, não fizeram distinção quanto a área de formação dos docentes, sendo o estudo E10 o único que propôs uma formação específica para professores de matemática e ciências. No trabalho de Campos e Libardoni (2020), que faz uma investigação da RE na educação brasileira (compreendendo o período de 1994 a 2016), percebe-se a predominância de estudos em três áreas: matemática, ciência e computação. Além disso, nos estudos cujo foco não estava na formação de professores e que, portanto, caíram em critério de exclusão, verificou-se que na maioria dos casos a robótica esteve associada ao ensino de matemática e ciências. Zilli (2004) afirma que através da robótica educacional é possível adquirir competências como raciocínio lógico, habilidades manuais e estéticas, relações interpessoais e intrapessoais, capacidade de desenvolvimento de projetos, investigação e compreensão. Assim sendo, percebe-se a importância de articular a robótica educacional com as demais áreas do conhecimento.

Quando analisada a questão "Quais níveis de educação básica costumam estar associados a projetos de robótica educacional?" pode-se afirmar que os trabalhos que descreveram a qual nível de educação básica os docentes estão associados, foi possível verificar que os anos iniciais e séries finais do ensino fundamental foram mais contemplados com as oficinas de robótica educacional.

Nesse caso, é importante ressaltar que muitos dos estudos apresentados, sobretudo os que não ocorreram no Brasil, dispuseram de uma estrutura que não condiz com o que se observa na rede pública de ensino brasileira. Nessas instituições estrangeiras havia espaço adequado e recursos para aquisição de materiais tais como, componentes eletrônicos, mecânicos e estruturais, motores, sensores e controladores programáveis.

Considerando o caso da escola pública brasileira esses recursos estão disponíveis somente em algumas delas, e a aquisição não é um processo simples, visto que é necessário submeter a compra a um processo de licitação, ou solicitar às secretarias (estaduais ou municipais) e aguardar a aquisição. Além disso, é necessário um software para programar o controlador, um computador que seja capaz de executar o programa escolhido e um ambiente físico específico, contendo elementos para interagir com o robô na realização de uma determinada tarefa (Neves, 2011).

Outro desafio identificado, durante a análise desses trabalhos, consiste na criação de dispositivos que estimulem os professores a incorporarem o uso da robótica 
educacional como parte de sua prática em sala de aula, o que justifica a preocupação encontrada em vários estudos na incorporação da robótica no currículo escolar.

Quando os estudos foram analisados sob a perspectiva da questão "Quais são as plataformas de prototipagem utilizadas com mais frequência nas escolas da rede pública de educação básica?" percebeu-se que eles se dividiram em três categorias: (i) oito estudos desenvolveram as pesquisas utilizando kits proprietários, sendo que os estudos internacionais utilizaram a plataforma Lego, já a investigação conduzida na Suíça utiliza a o kit Blue Bot $^{2}$; (ii) apenas duas investigações usaram plataforma Arduino, nas análises realizadas percebe-se que as formações estão mais direcionadas ao planejamento pedagógico e a estratégias que podem aplicar a robótica, e por isso o uso de kits Lego predomina nas formações realizadas; e (iii) apenas um estudo conduziu a formação de professores sem o uso de nenhum tipo de kit, concentrando-se em abordar questões metodológicas e pedagógicas vinculadas à robótica educacional.

No caso da categoria "Sem uso de kits" pode-se afirmar que o estudo E04 pode ser classificado como um estudo teórico. A pesquisa tem como alvo professores do ensino fundamental e médio que são alunos do Mestrado em Ensino de Informática ofertado em Portugal. $\mathrm{O}$ foco do trabalho compreende a análise dos níveis de interesse, conhecimento, resolução de problemas e autoconfiança dos professores na utilização de robótica educacional para desenvolver o pensamento computacional. Não apresenta proposta de método nem produto e tão pouco aponta a utilização de kits ou plataformas.

$\mathrm{Na}$ categoria kits open-source, ou robótica livre, relacionou-se apenas os estudos E05, E07, E08 e E10. O trabalho E05, embora utilize kits proprietários, também faz uso da ferramenta gratuita e open-source Scratch $\mathrm{Jr}^{3}$. Seguindo a mesma linha, o estudo E07 traz tanto a utilização de kits proprietários, como ferramentas open-source como o programa Scratch e a plataforma de prototipagem Arduino ${ }^{4}$ O estudo E08 apresentou o uso de materiais alternativos bem como a utilização de componentes eletrônicos com servo motores, LEDs e materiais reciclados. O estudo E10 aborda a criação e análise de uma formação semipresencial em robótica educacional para professores de Ciências e Matemática do município de Paracambi-RJ. O material empregado nas oficinas foi um kit de montagem com Arduino, sendo que este foi o único que utilizou somente kits opensource.

É possível verificar que, embora os kits baseados em robótica livre tragam um custo baixo para sua aquisição, estiveram presentes apenas em quatro dos dez estudos e, desses quatro estudos, apenas um não utilizou algum tipo de kit proprietários. Embora não esteja claro no corpo dos trabalhos os critérios utilizados na escolha dos kits, uma possível razão para a baixa utilização de ferramentas open-source é a necessidade de um conhecimento mais aprofundado de conhecimentos da área de Computação, tanto em linguagem de programação como em eletrônica e mecânica.

Na categoria kits proprietários, foram enquadrados os estudos E01, E02, E03, E05, E06, E07, E08 e E09, como descrito no Quadro 6.

\footnotetext{
${ }^{2}$ Disponível em: https://www.tts-international.com/blue-bot-bluetooth-programmable-floorrobot/1015269.html . O robô Blue-Bot é um robô programável que possui conexão wireless, podendo ser programando por botões ou por aplicativo que pode ser instalado tanto em tablets como em computadores.

${ }^{3}$ Disponível em: https://www.scratchjr.org/. Ele foi desenvolvido como uma colaboração entre a DevTech Research Group na Tufts University, a Lifelong Kindergarten Group no MIT Media Lab, e o Playful Invention Company.

${ }^{4}$ Arduino compreende um microcontrolador que pode ser programado com uma linguagem de programação baseada em $\mathrm{C} / \mathrm{C}++$, e é disponibilizado por meio da licença Creative Commons.
} 
Quadro 6. Comparativo Estudos com Kits Proprietários

\begin{tabular}{|c|c|c|c|c|}
\hline Estudo & Foco & Onde & Kit & Observações \\
\hline E01 & $\begin{array}{l}\text { Análise dos resultados } \\
\text { do curso de formação } \\
\text { para docentes. }\end{array}$ & 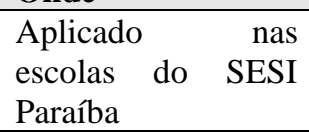 & $\begin{array}{l}\text { LEGO } \\
\text { Mindstorms } \\
\text { EV3 }^{5}\end{array}$ & \\
\hline E02 & $\begin{array}{l}\text { Apresenta análise de } \\
\text { um currículo para } \\
\text { formação de } \\
\text { professores. }\end{array}$ & $\begin{array}{l}\text { Realizado na } \\
\text { Europa no contexto } \\
\text { do projeto } \\
\text { ROBOESL }\end{array}$ & $\begin{array}{l}\text { LEGO } \\
\text { Mindstorms } \\
\text { EV3 }\end{array}$ & $\begin{array}{l}\text { Abordou como a RE pode } \\
\text { ser usada como recurso } \\
\text { para evitar o abandono } \\
\text { escolar. }\end{array}$ \\
\hline E03 & $\begin{array}{l}\text { Análise dos resultados } \\
\text { do curso de formação } \\
\text { para docentes }\end{array}$ & $\begin{array}{l}\text { Desenvolvido na } \\
\text { Itália }\end{array}$ & $\begin{array}{l}\text { LEGO } \\
\text { Mindstorms }\end{array}$ & \\
\hline E05 & $\begin{array}{l}\text { Apresenta um modelo } \\
\text { de currículo de } \\
\text { formação para } \\
\text { professores. }\end{array}$ & $\begin{array}{l}\text { Aplicado com } 350 \\
\text { docentes no Cantão } \\
\text { Vaud na Suiça }\end{array}$ & $\begin{array}{l}\text { Blue-Bot de } \\
\text { empresa TTS }\end{array}$ & $\begin{array}{l}\text { Integrar Ciências da } \\
\text { Computação com } \\
\text { Robótica educacional }\end{array}$ \\
\hline E06 & $\begin{array}{l}\text { Análise dos resultados } \\
\text { do curso de formação } \\
\text { para docentes. }\end{array}$ & $\begin{array}{l}\text { Aplicado em } 21 \\
\text { professores } \\
\text { americanos. }\end{array}$ & LEGO WeDo $^{6}$ & $\begin{array}{l}\text { Promover o ensino de } \\
\text { ciências, tecnologia, } \\
\text { engenharia e matemática } \\
\text { (Science, Technology, } \\
\text { Engineering and } \\
\text { Mathematics - STEM) }\end{array}$ \\
\hline E07 & $\begin{array}{l}\text { Análise dos resultados } \\
\text { do curso de formação } \\
\text { para docentes. }\end{array}$ & $\begin{array}{l}\text { Escola estadual } \\
\text { indígena em } \\
\text { Piraquara - Paraná }\end{array}$ & $\begin{array}{l}\text { Kits } \\
\text { proprietários } \\
\text { como LEGO e } \\
\text { Fischer } \\
\text { Technik }^{7}\end{array}$ & $\begin{array}{l}\text { Utilizou também a } \\
\text { plataforma Arduino para } \\
\text { demonstração de } \\
\text { materiais alternativos e o } \\
\text { Scratch para ensino de } \\
\text { programação por blocos. }\end{array}$ \\
\hline E08 & $\begin{array}{l}\text { Análise dos resultados } \\
\text { do curso de formação } \\
\text { para docentes. }\end{array}$ & $\begin{array}{l}\text { Professores do } \\
\text { ensino fundamental } \\
\text { da cidade de São } \\
\text { José dos Pinhais, } \\
\text { Paraná }\end{array}$ & $\begin{array}{l}\text { Robótica } \\
\text { Modelix } 27^{8}\end{array}$ & $\begin{array}{l}\text { Integrou componentes } \\
\text { eletrônicos }\end{array}$ \\
\hline E09 & $\begin{array}{l}\text { Análise do fazer } \\
\text { pedagógico de } \\
\text { professores egressos da } \\
\text { formação continuada } \\
\text { "Robótica Aplicada à } \\
\text { Educação" }\end{array}$ & $\begin{array}{l}\text { Aplicada em João } \\
\text { Pessoa - PB }\end{array}$ & $\begin{array}{l}\text { kits ALPHA } \\
\text { Mecatrônica }^{9}\end{array}$ & \\
\hline
\end{tabular}

\footnotetext{
${ }^{5}$ Kit que tem como característica peças que se encaixam e uma central programável tanto por software como por botões presentes na própria central.

${ }^{6}$ Disponível em: https://education.lego.com/en-us/products/lego-education-wedo-2-0-core-set/45300 . O kit LEGO WeDo possui como característica principal uma central simplificada com conexão via bluetooth compatível com sistema Android 4.4 ou superior.

${ }^{7}$ Disponível em: https://www.fischertechnik.de/en/products/teaching/stem-kits. O kit Fischer Technik possui uma central programável, motores e blocos encaixáveis.

${ }^{8}$ Disponível em:

https://www.modelix.com.br/?gclid=Cj0KCQjwkZiFBhD9ARIsAGxFX8Bv6bwoeM7HM-

0yjHJoBxOWr2ybPuKr_qs9tTw5BfueFrHO0NjUx74aApO1EALw_wcB . O kit de Robótica Modelix 27 conta com uma central programável e peças e engrenagens que se encaixam para formar estruturas.

${ }^{9}$ Disponível em: https://www.pete.com.br/kit-alpha-mecatronica . Os kits ALPHA Mecatrônica, desenvolvidos pela empresa PETE, apresentam variações que vão desde uma central com botões e LEDs para montagem de robô seguidor de linha, até centrais que podem ser programadas tanto em linguagem C como através de programação por blocos.
} 
De acordo com os dados encontrados é possível demonstrar que a maior parte dos estudos analisados utilizam kits e Ambientes Virtuais de Aprendizagem (AVAs) como recursos para o ensino de robótica. Não foi observado em nenhum dos estudos o uso de vídeo aulas para formação de professores. Dos kits utilizados, percebeu-se que a grande maioria são kits proprietários que, por trazerem parte do processo de construção/montagem do dispositivo robótico pronto, podem acabar por limitar o processo de aprendizagem e tem contribuições restritas ao desenvolvimento cognitivo dos estudantes. Para Chella (2002, p.23) a robótica educacional é

[...] um ambiente constituído pelo computador, componentes eletrônicos, eletromecânicos e programa, onde o aprendiz, por meio da integração desses elementos, constrói e programa dispositivos automatizados com o objetivo de explorar conceitos das diversas áreas do conhecimento.

Assim sendo, na utilização de kits onde a parte eletrônica e mecânica se resume a conectar fios e encaixar peças prontas, perde-se uma parte significativa do processo de construção do conhecimento, embora a interação pertinente à programação do objeto, também favoreça o desenvolvimento de algumas habilidades e do pensamento computacional.

Na nuvem de palavras esquematizada pela Figura 1, é possível observar em fonte maior as palavras que foram utilizadas com maior frequência nos estudos selecionados. É possível verificar que embora esses documentos tenham como foco a formação de professores, as palavras prática, alunos e estudantes aparecem repetidamente, demostrando assim uma preocupação com o resultado que tal formação pode trazer para os educandos. Além disso, as palavras "conhecimento" e "pesquisa" não apareceram com tanta ênfase, o que demonstra que as formações não se concentraram em abordar como a RE pode contribuir para o desenvolvimento da pesquisa e do conhecimento científico.

Figura 1. Nuvem de palavras

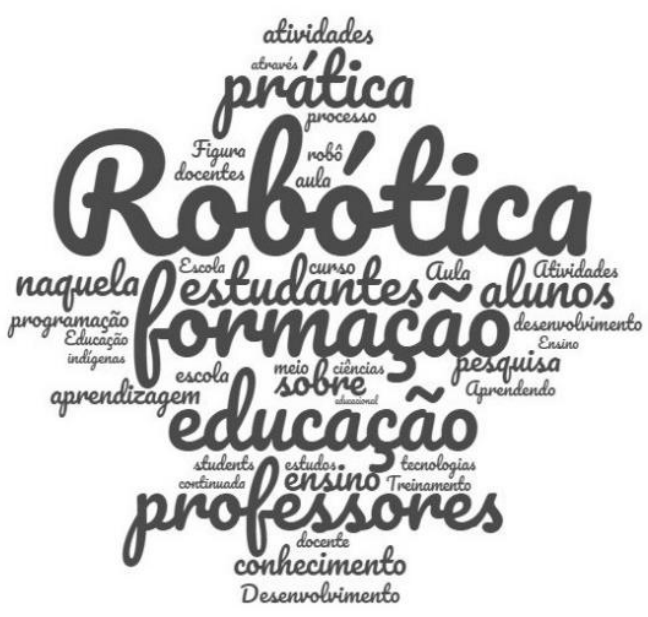

Fonte: Elaborada pelos autores (2021).

Pensando nas questões de pesquisa apresentadas anteriormente no Quadro 1, pode-se afirmar que essa RSL obteve as respostas das mesmas. Através da análise dos trabalhos é possível afirmar que as únicas formações direcionadas para as áreas de conhecimento dos docentes foram as que tiveram como centro o ensino de ciências. Percebeu-se também que os principais níveis de atuação dos professores foram os equivalentes aos anos finais do ensino fundamental. Foi possível também relacionar as 
ferramentas utilizadas nas formações conforme demonstrado no Quadro 6, assim como identificar que muitas das tecnologias utilizadas não são viáveis de serem aplicadas em escolas públicas, no contexto brasileiro, pois demandam investimentos financeiros que muitas não dispõem.

É importante ressaltar que embora os kits relacionados estejam presentes nos trabalhos dessa revisão da literatura, a robótica educacional não se resume meramente a utilização desses materiais. Muitos trabalhos observaram que a utilização desorientada desses recursos pode desmotivar o aluno e comprometer sua aprendizagem, e que é necessário utilizar teorias da aprendizagem para fundamentar a aplicação da RE em sala de aula (Campos; Libardoni, 2020).

\section{Considerações finais}

Este artigo buscou realizar um mapeamento sistemático da literatura aplicada à educação, proporcionando discutir e apresentar os estudos que estão sendo realizados sobre a formação de robótica educacional para docentes. Para tanto, foram estabelecidas questões de pesquisa que nortearam este mapeamento e critérios que resultaram na análise de dez estudos. Neste contexto, os trabalhos foram analisados visando responder as questões de pesquisa, facilitando a aquisição de parte do conhecimento científico acerca do tema investigado.

Após a execução desse estudo, é possível afirmar que a formação de professores para o uso de robótica educacional é uma preocupação que não se limita apenas ao Brasil. Contudo foi possível verificar também que a maior parte das propostas apresentadas não trazem a preocupação em ofertar uma alternativa de baixo custo. Observa-se a existência de uma preferência pela utilização de kits proprietários, desconsiderando a realidade de instituições que não possuem acesso a recursos que lhes permita tal aquisição.

De acordo com os resultados obtidos, percebe-se ainda a importância de um acompanhamento para verificar de que forma as formações ofertadas aos docentes impactam nos discentes. Esta preocupação que aparece presente na maioria dos estudos, aponta para a ideia de que o professor ensina o que aprende, necessitando assim de um programa de formação continuada que seja adequado a realidade da comunidade na qual ocorre sua prática.

Nessa perspectiva, observa-se uma escassez de estudos que discutam a aplicabilidade da robótica em sala de aula e usando recursos de baixo custo. Assim sendo, percebe-se que a presente pesquisa norteia possíveis desdobramentos para serem refletidos também sobre a formação inicial docente, tendo como foco disciplinas que abordam a utilização de recursos tecnológicos e como eles podem ser usadas nas práticas pedagógicas no contexto da sala de aula.

Com base no exposto, espera-se que o presente trabalho possa ser útil para aqueles que desejam ter uma visão geral sobre a formação de professores em robótica educacional, bem como possa servir como ponto de partida para futuros estudos. Além disso, salienta-se que ações isoladas têm obtido sucesso, mas que o ideal seria a implementação da RE no currículo escolar, visando potencializar, na educação básica, o desenvolvimento das habilidades apontadas na BNCC e do pensamento científico.

\section{Agradecimentos}

Este trabalho está vinculado a um projeto financiado pelo CNPq/IFRS. 


\section{Referências bibliográficas}

BRASIL. Base Nacional Comum Curricular. Brasília: MEC/Secretaria de Educação Básica, 2017. Disponível em: http://basenacionalcomum.mec.gov.br/. Acesso em: 15 ago. 2020.

CAMPOS, F. R.; LIBARDONI, G. C. Investigação em Robótica na Educação Brasileira: o que dizem as dissertações e teses. In: SILVA, R. B.; BLINKSTEIN, P. (Orgs.). Robótica Educacional: experiências inovadoras na educação brasileira. Porto Alegre: Penso, 2020. p. 21-45.

CHELlA, M. T. Ambiente de Robótica para Aplicações Educacionais com SuperLogo. 2002. Campinas: Universidade Estadual de Campinas - UNICAMP, Faculdade de Engenharia Elétrica e da Computação, 2002. Dissertação de Mestrado.

KITCHENHAM, B.A.; BRERETON, O.P.; BUDGEN, D. Using Mapping Studies as the Basis for Further Research - A Participant-Observer Case Study. Information and Software Technology, vol. 53, p. 638-651, 2011.

KITCHENHAM, B.A.; CHARTERS, S. Guidelines for performing systematic literature reviews in software engineering. Tech. Rep. EBSE-2007-01, Keele University, 2007.

NEVES, J. O. R. Desenvolvimento da fluência tecnológica em programa educacional de robótica pedagógica, 2011. Florianópolis: Universidade Federal de Santa Catarina, 2011. Dissertação de Mestrado.

PETERSEN, K.; FELDT, R.; MUJTABA, S.; MATTSSON, M. Systematic Mapping Studies in Software Engineering. In: International Conference on Evaluation and Assessment in Software Engineering, 8., 2008. Proceedings... p. 68-77, 2008.

RIBEIRO, C. R. Robô Carochinha: um estudo qualitativo sobre a robótica educativa no $1^{\circ}$ ciclo no ensino básico. 2006. Braga: Instituto de Educação e Psicologia, Universidade do Minho (Portugal), 2006. Dissertação de Mestrado em Educação Tecnologia Educativa.

SILVA, R. B.; BLIKSTEIN, P. Robótica Educacional: experiências inovadoras na educação brasileira. Porto Alegre: Penso, 2020. p. xix-xxiii.

ZILLI, S. R. A Robótica Educacional no Ensino Fundamental: perspectivas e prática. Florianópolis: Universidade Federal de Santa Catarina, 2004. Dissertação de Mestrado. 